Pacific Journal of Mathematics

ULTRASPHERICAL EXPANSIONS AND PSEUDO ANALYTIC

FUNCTIONS 


\title{
ULTRASPHERICAL EXPANSIONS AND PSEUDO ANALYTIC FUNCTIONS
}

\author{
ALLAN J. FRYANT
}

This paper takes up the function theoretic approach to the study of ultraspherical expansions, their conjugates, the associated elliptic equations, and first order systems. The theory of pseudo analytic functions and BergmanGilbert type integral operators are employed, and the relation between these two approaches is examined. Throughout, results obtained are analogs of well known theorems from the theory of analytic functions of a single complex variable, and the related study of harmonic functions and Fourier series.

The study of trigonometric series, analytic functions, Laplace's equation, and the Cauchy-Riemann system are all in a sense equivalent. Since this study has proven to be one of the most fruitful in mathematics, and since Laplace's equation is just one specific elliptic partial differential equation, analogous developments should be expected for more general elliptic equations. In particular, it is natural to hope for a relationship with analytic functions corresponding to that found in the case of harmonic functions, $u=\operatorname{Re}(f)$, which has proved so useful in the study of Laplace's equation and expansions in the associated special functions (trigonometric series).

For $u$ the solution of an elliptic equation more general than Laplace's, two approaches are apparent:

(1) Generalize the operation of "taking the real part". That is, find bounded linear operators which transform analytic functions to solutions $u$. Such results have been obtained, in particular, by Bergman [3] and Gilbert [14], where integral operators are developed to provide the transformation from analytic functions to solutions of corresponding elliptic equations.

(2) Generalize function theory. That is, extend function theory so that solutions $u$ of elliptic equations can be obtained as $u=\operatorname{Re}(f)$, where $f$ is a "pseudo" analytic function sharing many of the properties associated with classical analytic functions of a single complex variable. Bers [4], and Vekua [24] have developed such an approach.

In the pursuit of function theoretic results in this context, the case of ultraspherical expansions is of particular interest, since in this case both approaches (1) and (2) are directly applicable and intimately related. Using them, we obtain function theoretic results for ultraspherical expansions, adding to the extensive work of Muchenhaupt and Stein [19] on the subject. 
The ultraspherical (or Gegenbauer) polynomials $C_{n}^{\mu}(x), \mu>0$, of degree $n$ are defined by the generating relation

$$
\left(1-2 z x+z^{2}\right)^{-\mu}=\sum_{n=0}^{\infty} z^{n} C_{n}^{\mu}(x) .
$$

The sequence $\left\{C_{n}^{\prime \prime}(\cos \theta)\right\}_{n=0}^{\infty}$ is orthogonal and complete over $(0, \pi)$ with respect to the measure $\sin ^{2 \mu} \theta d \theta$. Thus a function $f(\theta)$ defined on $(0, \pi)$ has the expansion

$$
f(\theta) \sim \sum_{n=0}^{\infty} a_{n} C_{n}^{\mu}(\cos \theta),
$$

where

$$
\begin{aligned}
a_{n} & =\frac{\int_{0}^{\pi} f(\theta) C_{n}^{\mu}(\cos \theta) \sin ^{2 \mu} \theta d \theta}{\int_{0}^{\pi}\left[C_{n}^{\mu}(\cos \theta)\right]^{2} \sin ^{2 \mu} \theta d \theta} \\
& =\frac{2^{2 \mu-1} n !(n+\mu) \Gamma^{2}(\mu)}{\pi \Gamma(n+2 \mu)} \int_{0}^{\pi} f(\theta) C_{n}^{\mu}(\cos \theta) \sin ^{2 \mu} \theta d \theta .
\end{aligned}
$$

The Abel sums

$$
U(r, \theta)=\sum_{n=0}^{\infty} a_{n} r^{n} C_{n}^{\mu}(\cos \theta)
$$

of the series (1.2) arise as solutions of the partial differential equation

$$
U_{x x}+U_{y y}+\frac{2 \mu}{y} U_{y}=0,
$$

$(x, y)=(r \cos \theta, r \sin \theta)$, and are called generalized axisymmetric potentials (see [26] for a discussion of this equation and physical applications). Corresponding to equation (1.3) is the first order system

$$
\begin{aligned}
& U_{x}-V_{y}=\frac{2 \mu}{y} V \\
& U_{y}+V_{x}=0,
\end{aligned}
$$

defining the conjugate $V$ of $U[19$, p. 19]. $V$ satisfies the "conjugate equation"

$$
V_{x x}+V_{y y}+\frac{2 \mu}{y} V_{y}-\frac{2 \mu}{y^{2}} V=0 .
$$

Letting $u=U, v=y^{2 \mu} V$, if $U$ and $V$ satisfy (1.5), then, $u, v$ are solutions of 


$$
u_{x}=y^{-2 \mu} v_{y} \text { and } u_{y}=-y^{-2 \mu} v_{x} .
$$

If $U$ and $V$ are solutions of the equations (1.5), then $F=U+$ $i V$ is a pseudo analytic function of the first kind. We call such functions $\mu$-pseudo analytic. Since $\left[1, y^{-2 \mu}\right]$ is a generating pair for the system (1.5) the function $u+i v$ is the corresponding pseudo analytic function of the second kind (cf. the equations of mixed type example [9, pp. 389-390]). It is easy to show that if $U$ is a solution of equation (1.4) then there exists a function $V$ related to $U$ by the system (1.5), and $V$ is unique up to a function of $y$ alone. Specifically, given $U$ and $V$ satisfying (1.5), the functions $U$ and $V^{*}$ satisfy (1.5) if and only if $V^{*}(x, y)=V(x, y)+g(y)$, where $g$ satisfies $g^{\prime}+(2 \mu / y) g=0$. For convenience, if $U$ is given by the series expansion (1.3) following [2], [19] we choose as the conjugate $V$ related to $U$ by (1.5) the following:

$$
V(r, \theta)=2 \mu \sum_{n=1}^{\infty} \frac{1}{n+2 \mu} a_{n} r^{n} C_{n-1}^{\mu+1}(\cos \theta) \sin \theta .
$$

Gilbert [15] has developed an integral operator which tranforms analytic functions $f$ to generalized axisymmetric potentials $U$. If

$$
U(r, \theta)=\sum_{n=1}^{\infty} \frac{n !}{\Gamma(n+2 \mu)} a_{n} r^{n} C_{n}^{\prime \prime}(\cos \theta),
$$

then this $A_{\mu}$ operator gives

$$
\begin{aligned}
U(r, \theta) & =A_{\mu}(f) \\
& =\frac{\Gamma(2 \mu)}{2^{2 \mu-1} \Gamma^{2}(\mu)} \int_{0}^{\pi} f(r \cos \theta+i r \sin \theta \cos t) \sin ^{2 \mu-1} t d t,
\end{aligned}
$$

where $f(z)=\sum_{n=0}^{\infty} a_{n} z^{n}$. Heins [18] traces the origin of such integral representations back to Poisson [21]. An explicit integral representation for the inverse transform $A_{\mu}^{-1}$ was first obtained in [15], and is

$$
\begin{gathered}
f(z)=A_{\mu}^{-1}(U) \\
=\int_{0}^{\pi} U(r, t) K(z / r, \cos t) \sin t d t,|z|<r,
\end{gathered}
$$

where

$$
K(\sigma, \xi)=\frac{\Gamma^{2}(\mu+1)}{\pi} \frac{\left(1-\xi^{2}\right)^{\mu-1 / 2}\left(1-\sigma^{2}\right)}{\left(1-2 \xi \sigma+\sigma^{2}\right)^{\mu+1}} .
$$

Our first objective is to examine the relationship between the $A_{\mu}$ integral operator and $\mu$-pseudo analytic functions. Also, for $\mu=0,(1.4)$ and the conjugate equation (1.6) each reduce to Laplace's 
equation, and the system (1.5) becomes the Cauchy-Riemann system. Thus, given a proper formulation, all results regarding ultraspherical expansions, their conjugates, and $\mu$-pseudo analytic functions should in the limit as $\mu \rightarrow 0$ reduce to classical function theory.

2. Integral operator for the conjugate; the limit $\mu \rightarrow 0$. Throughout, we shall restrict attention to ultraspherical series having real coefficients. Since

$$
\lim _{\mu \rightarrow 0} \mu^{-1} C_{n}^{\mu}(x)=\frac{2}{n} T_{n}(x),
$$

where $T_{n}$ is the Tchebychev polynomial of degree $n$ (see [23, p. 178]), it is natural to renormalize the $A_{\mu}$ integral as follows: let

$$
L_{\mu}=\frac{2^{2 \mu-1}}{\mu \Gamma(2 \mu)} A_{\mu} .
$$

If

$$
U(r, \theta)=\frac{2^{2 \mu-1}}{\mu} \sum_{n=0}^{\infty} \frac{n}{\Gamma(n+2 \mu)} a_{n} r^{n} C_{n}^{\mu}(\cos \theta),
$$

then

$$
\begin{aligned}
U(r, \theta) & =L_{\mu}(f) \\
& =\frac{1}{\mu \Gamma^{2}(\mu)} \int_{0}^{\pi} f(r \cos \theta+i r \sin \theta \cos t) \sin ^{2 \mu-1} t d t,
\end{aligned}
$$

where

$$
f(z)=\sum_{n=0}^{\infty} a_{n} z^{n}
$$

We call $f$ the $L_{\mu}$ associate of $U$. The limit (2.1) then yields

$$
\begin{aligned}
\lim _{\mu \rightarrow 0} L_{\mu}\left(z^{n}\right) & =\lim _{\mu \rightarrow 0} \frac{2^{2 \mu-1} n !}{\mu \Gamma(n+2 \mu)} r^{n} C_{n}^{\mu}(\cos \theta) \\
& =r^{n} T_{n}(\cos \theta) \\
& =\operatorname{Re}\left(z^{n}\right) .
\end{aligned}
$$

Since $\left|L_{\mu}\left(z^{n}\right)-\operatorname{Re}\left(z^{n}\right)\right| \sim g(n)$ on $|z| \leqq 1$, where $[g(n)]^{1 / n} \rightarrow 1$ as $n \rightarrow \infty$, we have the following

THEOREM 2.1. Let $f(z)=\sum_{n=0}^{\infty} a_{n} z^{n}$, where $a_{n}$ are real, and $\varlimsup\left|a_{n}\right|^{1 / n}=1 / R, R>1$. Then

$$
\lim _{\mu \rightarrow 0} L_{\mu}(f)=\operatorname{Re}(f)
$$


uniformly on $|z| \leqq 1$.

Proof.

$$
\left|L_{\mu}(f)-\operatorname{Re}(f)\right| \leqq \sum_{n=0}^{\infty}\left|\frac{2^{2 \mu-1} n !}{\mu \Gamma(n+2 \mu)} C_{n}^{\mu}(\cos \theta)-\cos n \theta\right|\left|a_{n}\right| r^{{ }^{n}}
$$

By the result [23, p. 182]

$$
C_{n}^{\mu}(\cos \theta)=\sum_{m=0}^{n} \alpha_{m} \alpha_{n-m} \cos (n-2 m) \theta,
$$

where

$$
\alpha_{m}=\frac{\Gamma(\mu+m)}{\Gamma(\mu) m !}
$$

It is easy to see that

$$
\lim _{\mu \rightarrow 0} \frac{\alpha_{m} \alpha_{n-m}}{\mu}= \begin{cases}0 & \text { if } m \neq 0, n \\ 1 / n & \text { if } m=0, n .\end{cases}
$$

Thus

$$
\begin{aligned}
& \left|\frac{2^{2 \mu-1} n !}{\mu \Gamma(n+2 \mu)} C_{n}^{\mu}(\cos \theta)-\cos n \theta\right| \\
& \leqq\left|\frac{2^{2 \mu-1} n !}{\mu \Gamma(n+2 \mu)} C_{n}^{\mu}(\cos \theta)-\frac{2^{2 \mu} n !}{\mu \Gamma(n+2 \mu)} \alpha_{0} \alpha_{n} \cos n \theta\right| \\
& +\left|\frac{2^{2 \mu} n !}{\mu \Gamma(n+2 \mu)} \alpha_{0} \alpha_{n} \cos n \theta-\cos n \theta\right| \\
& \leqq \frac{2^{2 \mu-1}}{\mu} \sum_{m=1}^{n-1} \frac{n !}{\Gamma(n+2 \mu)} \alpha_{m} \alpha_{n-m}+\left|\frac{2^{2 \mu} n ! \alpha_{0} \alpha_{n}}{\mu \Gamma(n+2 \mu)}-1\right|,
\end{aligned}
$$

and for $0<\mu<1$, this is

$$
\leqq n^{2}\left[\frac{2^{2 \mu-1}}{\mu \Gamma^{2}(\mu)}+\frac{1}{n^{2}}\left|\frac{2^{2 \mu} \Gamma(n+\mu)}{\mu \Gamma(\mu) \Gamma(n+2 \mu)}-1\right|\right] .
$$

Now, since $\Gamma(n+\mu) / \Gamma(n+2 \mu) \sim(n+\mu)^{-\mu}$ as $n \rightarrow \infty$, and as $\mu \rightarrow 0$, $\mu \Gamma(\mu) \rightarrow 1$, there exists an $N_{1}$ such that

$$
\frac{2^{2 \mu-1}}{\mu \Gamma^{2}(\mu)}+\frac{1}{n^{2}}\left|\frac{2^{2 \mu} \Gamma(n+\mu)}{\mu \Gamma(\mu) \Gamma(n+2 \mu)}-1\right| \leqq c,
$$

for all $n>N_{1}$ and all $\mu \in[0,1]$, where $c$ is a constant (eg., 3 will do). Thus given $\varepsilon>0$, there exists an $N_{2}>N_{1}$ such that

$$
\sum_{n=N_{2}}^{\infty}\left|\frac{2^{2 \mu-1} n !}{\mu \Gamma(n+2 \mu)} C_{n}^{\mu}(\cos \theta)-\cos n \theta\right|\left|a_{n}\right| r^{n}<\varepsilon
$$


for all $\mu \in[0,1], \theta \in[0, \pi]$, and $0 \leqq r \leqq 1$. The convergence (2.4) of the first $N_{2}$ terms then completes the proof.

The conjugate $V$ of $U$ given by (2.2) has the series expansion

$$
V(r, \theta)=2^{2 \mu} \sum_{n=1}^{\infty} \frac{n !}{\Gamma(n+2 \mu+1)} a_{n} r^{n} C_{n-1}^{\mu+1}(\cos \theta) \sin \theta .
$$

Bers and Gelbart [8, p. 177] have given an integral representation for conjugate functions which yields the definition of a "conjugate" integral operator $L_{\mu}^{*}$ that transforms the $L_{\mu}$ associate $f$ of $U$ to $V$. Appealing to this result, the following is immediate:

If $U$ is given by the series (2.2), then a function connected with $U$ by the equations (1.5) is

$$
V(r, \theta)=-\frac{i}{\mu \Gamma^{2}(\mu)} \int_{0}^{\pi} f(r \cos \theta+i r \sin \theta \cos t) \cos t \sin ^{2 \mu-1} t d t .
$$

We further verify directly that this expression has the series expansion (2.5) above. An integration by parts shows that

$$
\begin{aligned}
& -\frac{i}{\mu \Gamma^{2}(\mu)} \int_{0}^{\pi}(r \cos \theta+i r \sin \theta \cos t)^{n} \cos t \sin ^{2 \mu-1} t d t \\
& \quad=\frac{n r \sin \theta}{2 \Gamma^{2}(\mu+1)} \int_{0}^{\pi}(r \cos \theta+i r \sin \theta \cos t)^{n-1} \sin ^{2 \mu+1} t d t \\
& \quad=\frac{n}{2}(\mu+1) r \sin \theta L_{\mu+1}\left(z^{n-1}\right) \\
& =\frac{2^{2 \mu} n !}{\Gamma(n+2 \mu+1)} r^{n} C_{n-1}^{\mu+1}(\cos \theta) \sin \theta,
\end{aligned}
$$

which yields the result. Also, since

$$
\left|C_{n}^{\prime \prime}(x)\right| \leqq \frac{\Gamma(n+2 \mu)}{n ! \Gamma(2 \mu)},
$$

the three series (2.2), (2.3), and (2.5) all have the same radius of convergence, i.e., they all converge absolutely and uniformly on compact subsets of $|z|<R$, where $\varlimsup\left|a_{n}\right|^{1 / n}=R^{-1}$. Thus, defining

$$
L_{\mu}^{*}(f)=-\frac{i}{\mu \Gamma^{2}(\mu)} \int_{0}^{\pi} f(r \cos \theta+i r \sin \theta \sin t) \cos t \sin ^{2 \mu-1} t d t,
$$

on interchanging the order of summation and integration we find that if $f$ has the Taylor series expansion (2.3), then $L_{\mu}^{*}(f)$ is given by the series (2.5). (Regarding the conjugate operator, see also [14, p. 189]). Further, the $L_{\mu}$ and $L_{\mu}^{*}$ integral representations for $U$ and $V$ obtain throughout the common disk $|z|<R$.

In view of Theorem 1 , it is natural to expect that $L_{\mu}^{*}(f) \rightarrow \operatorname{Im}(f)$ 
as $\mu \rightarrow 0 . \quad$ Now,

$$
\lim _{: \because 0} C_{n-1}^{n+1}(\cos \theta)=C_{n-1}^{1}(\cos \theta)=\frac{\sin n \theta}{\sin \theta}
$$

(see [23, p. 187]). Thus

$$
\begin{aligned}
\lim _{n \rightarrow 0} L_{!^{*}}^{*}\left(z^{n}\right) & =\lim _{n \rightarrow 0} \frac{2^{2 \mu} n !}{\Gamma(n+2 \mu+1)} a_{n} C_{n-1}^{\prime \prime+1}(\cos \theta) \sin \theta \\
& =r^{n} \sin n \theta \\
& =\operatorname{Im}\left(z^{n}\right) .
\end{aligned}
$$

Again, since $\left|L_{\mu}^{*}\left(z^{n}\right)-\operatorname{Im}\left(z^{n}\right)\right| \sim g(n)$ on $|z| \leqq 1$, where $[g(n)]^{1 / n} \rightarrow 1$ as $n \rightarrow \infty$, we have the desired result for functions which are analytic on a closed disk:

THEOREM 2.2. Let $f(z)=\sum_{n=0}^{\infty} a_{n} z^{n}$, where $a_{n}$ are real and $\varlimsup_{n \rightarrow \infty}\left|a_{n}\right|^{1 / n}=1 / R, R>1$. Then

$$
\lim _{\mu \rightarrow 0} L_{\mu}^{*}(f)=\operatorname{Im}(f)
$$

uniformly on $|z| \leqq 1$.

Proof. The generating relation (1.1) yields

$$
\frac{z \sin \theta}{\left(1-2 z \cos \theta+z^{2}\right)^{\mu+1}}=\sum_{n=1}^{\infty} z^{n} C_{n-1}^{\mu+1}(\cos \theta) \sin \theta
$$

Subtracting

$$
\frac{z \sin \theta}{1-2 z \cos \theta+z^{2}}=\sum_{n=1}^{\infty} z^{n} \sin n \theta
$$

and noting that each series converges uniformly on compact subsets of $|z|<1$, we have for $0<\rho<1, n=1,2, \cdots$,

$$
\begin{aligned}
& C_{n-1}^{\prime \mu+1}(\cos \theta)-\sin n \theta \\
& \quad=\frac{1}{2 \pi i} \int_{|z|=\rho} \frac{z \sin \theta\left[1-\left(1-2 z \cos \theta+z^{2}\right)^{\mu}\right]}{\left(1-2 z \cos \theta+z^{2}\right)^{\mu+1}} \frac{d z}{z^{n+1}} .
\end{aligned}
$$

Thus

$$
\left|C_{n-1}^{\mu+1}(\cos \theta) \sin \phi-\sin n \theta\right| \leqq \frac{C}{\rho^{n-1}}
$$

for all $\theta \in[0,2 \pi]$, and $\mu \in[0,1]$, where $C$ is a constant depending only on $\rho$. Now, 


$$
\begin{aligned}
\mid L^{*}(f) & -\operatorname{Im}(f) \mid \\
\leqq & \sum_{n=1}^{\infty}\left|\frac{2^{2 \mu} n !}{\Gamma(n+2 \mu+1)} a_{n} r^{n} C_{n-1}^{\mu+1}(\cos \theta) \sin \theta-a_{n} r^{n} \sin n \theta\right| \\
\leqq & \sum_{n=1}^{\infty}\left|a_{n}\right| r^{n}\left[\mid \frac{2^{2 \mu} n !}{\Gamma(n+2 \mu+1)} C_{n-1}^{\mu+1}(\cos \theta) \sin \theta\right. \\
& \left.\quad-C_{n-1}^{\mu+1}(\cos \theta) \sin \theta|+| C_{n-1}^{\mu+1}(\cos \theta) \sin \theta-\sin n \theta \mid\right] .
\end{aligned}
$$

Let $\varepsilon>0$. Choose $\rho \in(0,1)$ sufficiently near 1 so that $\sum_{n=0}^{\infty}\left|a_{n}\right| \rho^{-n}$ converges. Then by the result (2.10), there exists an $N_{1}$ such that

$$
\sum_{n=N_{1}}^{\infty}\left|a_{n}\right| r^{n}\left|C_{n-1}^{\mu+1}(\cos \theta) \sin \theta-\sin n \theta\right|<\varepsilon
$$

for all $r \in[0,1], \theta \in[0,2 \pi]$. Further, using the bound (2.6), we have

$$
\begin{aligned}
& \left|\frac{2^{2 \mu} n !}{\Gamma(n+2 \mu+1)} C_{n-1}^{\mu+1}(\cos \theta) \sin \theta-C_{n-1}^{\mu+1}(\cos \theta) \sin \theta\right| \\
& \leqq\left|\frac{2^{2 \mu} n !}{\Gamma(n+2 \mu+1)}-1\right| \frac{\Gamma(n+2 \mu+1)}{(n-1) ! \Gamma(n+2 \mu)} \\
& \leqq \frac{\left(2^{2 \mu}+1\right)(n+1)(n+2)}{\Gamma(2 \mu+2)} .
\end{aligned}
$$

Thus there exists an $N_{2}$ such that

$$
\sum_{n=N_{2}}^{\infty}\left|a_{n}\right| r^{n}\left|\frac{2^{2 \mu} n !}{\Gamma(n+2 \mu+1)} C_{n-1}^{\mu+1}(\cos \theta) \sin \theta-C_{n-1}^{\mu+1}(\cos \theta) \sin \theta\right|<\varepsilon .
$$

The convergence (2.8) of the first $N=\max \left(N_{1}, N_{2}\right)$ terms then completes the proof.

Define

$$
\mathscr{L}_{\mu}=L_{\mu}+i L_{\mu}^{*}
$$

If $U$ and $V$ are conjugate functions given by (2.2) and (2.5), then $F=U+i V$ is a $\mu$-pseudo analytic function of the first kind, and

$$
\begin{aligned}
F(r, \theta) & =\mathscr{L}_{\mu}(f) \\
& =\frac{1}{\mu \Gamma^{2}(\mu)} \int_{0}^{\pi} f(r \cos \theta+i r \sin \theta \cos t)(1+\cos t) \sin ^{2 \mu-1} t d t,
\end{aligned}
$$

where $f(z)=\sum_{n=0}^{\infty} a_{n} z^{n}$. If $F$ is regular on $|z|<1$ (i.e., $U$ and $V$ are $C^{2}$ solutions of the system (1.5)), then the $\mathscr{L}_{\mu}$ associate $f$ of $F$ is analytic on $|z|<1$, and the integral representation (2.11) holds throughout the $\operatorname{disk}|z|<1$.

As an immediate consequence of Theorems 1 and 2, we have the 
COROLLARY. Let $f(z)=\sum_{n=0}^{\infty} a_{n} z^{n}$, where $a_{n}$ are real, and $\varlimsup\left|a_{n}\right|^{1 / n}=1 / R, R>1$. Then

$$
\lim _{\mu \rightarrow 0} \mathscr{L}_{\mu}(f)=f
$$

uniformly on $|z| \leqq 1$.

Thus analytic functions of a single complex variable appear as a limiting case of $\mu$-pseudo analytic functions.

With the exception of an explicit representation for the inverse transform $\left(L_{\mu}^{*}\right)^{-1}$, our discussion of integral operators which generalize the operations $R e$ and $I m$, and examination of their relationship with pseudo analytic functions is complete. The development of an explicit representation for $\left(L_{*}^{*}\right)^{-1}$ will be postponed to the next section, since (as will be shown) both this transform and $L_{\mu}^{-1}$ are closely related to the Poisson integral.

3. Function theoretic results on a disk. Since ultraspherical expansions and their conjugates enjoy the same relation with $\mu$-pseudo analytic functions that is found between trigonometric series and analytic functions, it is natural to expect analogs of classical function theoretic results on a disk. Such results have been obtained by Muckenhaupt and Stein [19] and include, in particular, a Poisson integral formula for $U$, conjugate kernel, analog of Fatou's theorem, Riese's theorem $(U \rightarrow V$ is a bounded operator on $L^{p}$ ), and $H^{p}$ theory. Our objectives in this section are to examine the relationship between the Poisson integral formula and the transform $L_{\mu}^{-1}$, obtain new integral representations for the Poisson kernel and conjugate kernel, develop an explicit integral representation for $\left(L_{*}^{*}\right)^{-1}$, and to give an extension of Privaloff's theorem [20] to ultraspherical expansions and their conjugates.

If $U(\theta)$ has ultraspherical expansion

$$
U(\theta)=\frac{2^{2 \mu-1}}{\mu} \sum_{n=0}^{\infty} \frac{n !}{\Gamma(n+2 \mu)} a_{n} C_{n}^{\mu}(\cos \theta),
$$

then the Abel sum of this series

$$
U(r, \theta)=\frac{2^{2 \mu-1}}{\mu} \sum_{n=0}^{\infty} \frac{n !}{\Gamma(n+2 \mu)} a_{n} r^{n} C_{n}(\cos \theta)
$$

is given by the "Poisson integral"

$$
U(r, \theta)=\int_{0}^{\pi} U(\phi) P(r, \theta, \phi) \sin ^{2 \mu} \phi d \phi,
$$

where the "Poisson kernel" $P$ is 


$$
\begin{aligned}
& P(r, \theta, \phi)=\frac{\Gamma^{2}(\mu) 2^{2 \mu-1}}{\pi} \sum_{n=0}^{\infty} \frac{(n+\mu) n !}{\Gamma(n+2 \mu)} r^{n} C_{n}^{\mu}(\cos \theta) C_{n}^{\mu}(\cos \phi) \\
& =\frac{\mu\left(1-r^{2}\right)}{\pi} \int_{0}^{\pi} \frac{\sin ^{2 \mu-1} t}{\left[1-2 r(\cos \theta \cos \phi+\sin \theta \sin \phi \cos t)+r^{2}\right]^{\mu+1}} d t
\end{aligned}
$$

(see $\left[19\right.$, p. 25]). Gilbert's inverse transform $L_{\mu}^{-1}$ is easily obtained from the Poisson integral formula (3.2). Letting $\theta=0$ in (3.2) and analytically continuing $v$ to complex values $z$ throughout the disk $|z|<1$ yields

$$
U(z, 0)=\int_{0}^{\pi} U(\phi) P(z, 0, \phi) \sin ^{2 \mu} \phi d \phi,
$$

where

$$
\begin{aligned}
P(z, 0, \phi) & =\frac{\mu}{\pi} \frac{1-z^{2}}{\left(1-2 z \cos \phi+z^{2}\right)^{\mu+1}} \int_{0}^{\pi} \sin ^{2 \mu-1} t d t \\
& =\frac{\mu \Gamma(\mu) \Gamma(1 / 2)}{\pi \Gamma(\mu+1 / 2)} \frac{1-z^{2}}{\left(1-2 z \cos \phi+z^{2}\right)^{\mu+1}} .
\end{aligned}
$$

Since $C_{n}^{\mu}(1)=\Gamma(n+2 \mu) / n ! \Gamma(2 \mu)$, we have

$$
U(z, 0)=\frac{2^{2 \mu-1}}{\mu \Gamma(2 \mu)} \sum_{n=0}^{\infty} a_{n} z^{n}=\frac{2^{2 \mu+1}}{\mu \Gamma(2 \mu)} f(z),
$$

where $f$ is the $L_{\mu}$ associate of $U(r, \theta)$. Thus (3.3) yields for $|z|<1$,

$$
f(z)=\frac{\Gamma^{2}(\mu+1)}{\pi} \int_{0}^{\pi} U(\phi) \frac{1-z^{2}}{\left(1-2 z \cos \phi+z^{2}\right)^{\mu+1}} \sin ^{2 \mu} \phi d \phi,
$$

which is the expression (1.9) for $L_{\mu}^{-1}$ in the case where the integral is taken over the unit half circle.

Conversely, one obtains the Poisson integral formula by applying the $L_{\mu}$ transform to the expression for $L_{\mu^{-1}}^{-1}$ Letting $r=1$ in (1.9), we have for $|z|<1$

$$
\begin{gathered}
L_{\mu}[f(z)]=L_{\mu}\left[\frac{\Gamma^{2}(\mu+1)}{\pi} \int_{0}^{\pi} U(1, \phi) \frac{1-z^{2}}{\left(1-2 z \cos \phi+z^{2}\right)^{\mu+1}} \sin ^{2 \mu} \phi d \phi\right. \\
=\frac{\Gamma^{2}(\mu+1)}{\pi} \int_{0}^{\pi} U(1, \phi) L_{\mu}\left[\frac{1-z^{2}}{\left(1-2 z \cos \phi+z^{2}\right)^{\mu+1}}\right] \sin ^{2 \mu} \dot{\phi} d \dot{\phi} .
\end{gathered}
$$

Now,

$$
\frac{1-z^{2}}{\left(1-2 z \cos \phi+z^{2}\right)^{\mu+1}}=\sum_{n=0}^{\infty} \frac{n+\mu}{\mu} z^{n} C_{n}^{\mu}(\cos \phi),
$$

and the series converges uniformly for $|z| \leqq \rho<1, \phi \in[0, \pi]$. Applying the $L_{\mu}$ transform termwise to this series yields 


$$
\begin{aligned}
L_{\mu}\left[\frac{1-z^{2}}{\left(1-2 z \cos \phi+z^{2}\right)^{\mu+1}}\right] & =\frac{2^{2 \mu-1}}{\mu^{2}} \sum_{n=0}^{\infty} \frac{(n+\mu) n !}{\Gamma(n+2 \mu)} r^{n} C_{n}^{\mu}(\cos \phi) C_{n}^{\mu}(\cos \theta) \\
& =\frac{\pi}{\Gamma^{2}(\mu+1)} P(r, \theta, \phi) .
\end{aligned}
$$

Since $U(r, \theta)=L_{\mu}[f(z)],(3.4)$ is the Poisson integral formula for $U$. Note that this also yields an additional integral representation for the Poisson kernel:

$$
\begin{aligned}
& P(r, \theta, \phi) \\
& \quad=\frac{\mu}{\pi} \int_{0}^{\pi} \frac{1-(x+i y \cos t)^{2}}{\left[1-2(x+i y \cos t) \cos \phi+(x+i y \cos t)^{2}\right]^{\mu+1}} \sin ^{2 \mu-1} t d t,
\end{aligned}
$$

where $(x, y)=(r \cos \theta, r \sin \theta)$.

Again let $U(r, \theta)$ be given by the series (3.1). Then the conjugate $V(r, \theta)$ of $U$ has on the unit disk the representation

$$
V(r, \theta)=\int_{0}^{\pi} U(1, \phi) Q(r, \theta, \phi) \sin ^{2 \mu} \phi d \phi,
$$

where $Q$ is the "conjugate Poisson kernel"

$$
Q(r, \theta, \phi)=\frac{2^{2 \mu} \mu \Gamma^{2}(\mu)}{\pi} \sum_{n=0}^{\infty} \frac{n !(n+\mu)}{\Gamma(n+2 \mu+1)} r^{n} C_{n}^{\mu}(\cos \phi) C_{n-1}^{\mu+1}(\cos \theta) \sin \theta
$$

(see $\left[19\right.$, p. 35]). On applying the $L_{\mu}^{*}$ transform to the generating relation (3.5), a routine computation yields an integral representation for $Q$ similar to that found above for $P$ :

$$
Q(r, \theta, \phi)
$$

$$
=\frac{-i \mu}{\pi} \int_{0}^{\pi} \frac{1-(x+i y \cos t)}{\left[1-2(x+i y \cos t) \cos \phi+(x+i y \cos t)^{2}\right]^{\mu+1}} \cos t \sin ^{2 \mu-1} t d t .
$$

Expressed in terms of the $L_{\mu}, L_{\mu}^{*}$ integral operators, the representations (3.6) and (3.7) become

$$
P(r, \theta, \phi)=\frac{\Gamma(\mu+1)}{\pi} L_{\mu}\left[\frac{1-z^{2}}{\left(1-2 z \cos \phi+z^{2}\right)^{\mu+1}}\right],
$$

and

$$
Q(r, \theta, \phi)=\frac{\Gamma(\mu+1)}{\pi} L_{\mu}^{*}\left[\frac{1-z^{2}}{\left(1-2 z \cos \phi+z^{2}\right)^{\mu+1}}\right] .
$$

Thus, for $0 \leqq r<1, \theta \in[0,2 \pi]$,

$$
F(r, \theta)=\int_{0}^{\pi} U(1, \phi)[P(r, \theta, \phi)+i Q(r, \theta, \phi)] \sin ^{2 \mu} \phi d \phi,
$$

where $F=U+i V$, and 


$$
P(r, \theta, \dot{\phi})+i Q(r, \theta, \dot{\phi})=\frac{\Gamma(\mu+1)}{\pi} \mathscr{L}_{\mu}\left[\frac{1-z^{2}}{\left(1-2 z \cos \phi+z^{2}\right)^{\mu+1}}\right] .
$$

This result provides an analog of the Schwartz formula

$$
f(z)=\frac{1}{2 \pi} \int_{0}^{2 \pi} u\left(e^{2 t}\right) \frac{e^{i t}+z}{e^{i t}-z} d t
$$

which recovers an analytic function $f$ from its real part $u$. Notice that on restricting $\theta=0$ in (3.8) and analytically continuing $r$ to complex values $\zeta$ in $|\zeta|<1$, the integral reduces to the expression for the inverse transform $L_{\mu}^{-1}$. This integral representation for $L^{-1}$ might also be viewed as analogous to Schwartz's formula. For $L_{\mu}$ provides a transformation from analytic functions to solutions of an elliptic equation - in fact, is a continuous extension of the operation Re. And Schwartz's formula is nothing more than an explicit integral representation for the transform $\mathrm{Re}^{-1}$. This observation leads to the following analog of Privaloff's theorem [20]:

THEOREM 3. If

$$
U(\theta)=\frac{2^{2 \mu-1}}{\mu} \sum_{n=0}^{\infty} \frac{n !}{\Gamma(n+2 \mu)} a_{n} C_{n}^{\prime \prime}(\cos \theta)
$$

satisfies a Lipschitz condition of order $\alpha, 0<\mu<\alpha<1$, on $[0, \pi]$, then its conjugate

$$
V(\theta)=2^{2, \prime} \sum_{n=0}^{\infty} \frac{n !}{\Gamma(n+2 \mu+1)} a_{n} C_{n-1}^{\prime \prime+1}(\cos \theta) \sin \theta
$$

satisfies a Lipschizt condition of order $\alpha-\mu$ on $[0, \pi]$.

Proof. Hardy and Littlewood [17] have shown the following: an analytic function $f(z)$ satisfies $\left|f^{\prime}(z)\right|<C /(1-|z|)^{1-\alpha}$ throughout the disk $|z|<1$ if and only if $f$ satisfies a Lipschitz condition of order $\alpha$ on $|z| \leqq 1$. On applying this result to the Schwartz formula, a proof of the classical theorem of Privaloff obtains (see $[16$, p. 414]). In an entirely similar manner, differentiating the expression (3.3a) for $L_{v^{-1}}$ and appealing to the result of Hardy and Littlewood one obtains a Lipschitz condition of order $\alpha-\mu$ on the $L_{\mu}$ associate $f$ of $U$ (for details see [12]). That is, for $f(z)=$ $\sum_{n=0}^{\infty} a_{n} z^{n}$ we have

$$
\left|f\left(z_{1}\right)-f\left(z_{2}\right)\right| \leqq K\left|z_{1}-z_{2}\right|^{\alpha-\iota^{\prime \prime}}\left|z_{1}\right|,\left|z_{2}\right|<1 .
$$

Since 


$$
\begin{aligned}
V(r, \theta) & =L_{\mu}^{*}(f) \\
& =-\frac{i}{\mu \Gamma^{2}(\mu)} \int_{0}^{\pi} f(r \cos \theta+i r \sin \theta \cos t) \cos t \sin ^{2 \mu-1} t d t,
\end{aligned}
$$

it is then easy to see that for $0 \leqq r_{1}, r_{2} \leqq 1$,

$$
\begin{gathered}
\left|V\left(r_{1}, \theta_{1}\right)-V\left(r_{2}, \theta_{2}\right)\right| \leqq \frac{\pi K}{\Gamma^{2}(\mu+1)}\left[\left(r_{1} \cos \theta_{1}-r_{2} \cos \theta_{2}\right)^{2}\right. \\
\left.+\left(r_{1} \sin \theta_{1}-r_{2} \sin \theta_{2}\right)^{2}\right]^{(\alpha-\mu) / 2}
\end{gathered}
$$

In particular,

$$
\left|V\left(1, \theta_{1}\right)-V\left(1, \theta_{2}\right)\right| \leqq \frac{\pi K}{\Gamma^{2}(\mu+1)}\left|\theta_{1}-\theta_{2}\right|^{\alpha-\mu} .
$$

CoROllary. Let $F(z)=U(r, \theta)+i V(r, \theta)$ be $\mu$-pseudoanalytic on the open disk $|z|<1$. If $U$ is continuous on the closed disk $|z| \leqq$ 1 , and

$$
\left|U\left(1, \theta_{1}\right)-U\left(1, \theta_{2}\right)\right| \leqq K\left|\theta_{1}-\theta_{2}\right|^{\alpha}, 0<\mu<\alpha<1,
$$

then $F$ is continuous on the closed disk $|z| \leqq 1$, there satisfying a Lipschitz condition of order $\alpha-\mu$, i.e.,

$$
\left|F\left(z_{1}\right)-F\left(z_{2}\right)\right| \leqq M\left|z_{1}-z_{2}\right|^{\alpha-\mu}, \quad\left|z_{1}\right|,\left|z_{2}\right| \leqq 1
$$

Proof. As in the proof of the previous theorem, the hypotheses on $U$ imply that the $L_{\mu}$ associate $f$ of $U$ satisfies a Lipschitz condition of order $\alpha-\mu$ on the closed disk $|z| \leqq 1$. Since

$$
F(z)=\mathscr{L}_{\mu}(f)=\frac{1}{\mu \Gamma^{2}(\mu)} \int_{0}^{\pi} f(x+i y \cos t)(1+\cos t) \sin ^{2 \mu-1} t d t,
$$

the result is immediate.

Let $U(r, \theta)$ be given by the series (3.1), where $\left|a_{n}\right|^{1 / n} \rightarrow 1$ as $n \rightarrow \infty$. In [12] it was shown that if, further, on the boundary circle $U$ satisfies

$$
\left|U\left(1, \theta_{1}\right)-U\left(1, \theta_{2}\right)\right| \leqq \lambda\left(\left|\theta_{1}-\theta_{2}\right|\right),
$$

where

$$
\int_{0}^{\pi / 2} \frac{\lambda(t)}{t^{\mu+1}} d t<\infty
$$

then $f(z)=L_{\mu}^{-1}(U)$ is bounded on $|z| \leqq 1$. Thus $F=\mathscr{L}_{\mu}(f)$ is bounded on $|z| \leqq 1$, and we see that condition (3.10) is sufficient to insure that the conjugate $V$ of $U$ is bounded on $|z| \leqq 1$. We note that this result, as well as those of the above theorem and corollary, 
reduce in the limit as $\mu \rightarrow 0$ to classical function theoretic results which are known to be best possible.

It is also possible to express $U$ interior to a disk in terms of an integral of its conjugate $V$ over the boundary circle. Such a result leads immediately to an explicit integral formula for the transform $\left(L_{\mu}^{*}\right)^{-1}$. Define

$$
\begin{gathered}
Q^{*}(r, \theta, \phi)=\frac{2^{2 \mu} \Gamma^{2}(\mu+1)}{\pi \mu} \sum_{n=1}^{\infty} \frac{(n+\mu)(n-1) !}{\Gamma(n+2 \mu)} \\
\cdot r^{n} C_{n}^{\mu}(\cos \theta) C_{n-1}^{\mu+1}(\cos \phi) \sin \phi
\end{gathered}
$$

Then for all $r \in[0,1), \theta \in[0,2 \pi]$, we have

$$
U(r, \theta)=\int_{0}^{\pi} V(1, \phi) Q^{*}(r, \theta, \phi) \sin ^{2 \mu} \phi d \phi+U_{0},
$$

where $U_{0}=U(0, \theta)$. This result is easily obtained by termwise integration of the series expansion (2.5) for $V$. Now, restricting $\theta=0$ in this expression and analytically continuing $r$ to complex values $z,|z|<1$, yields

$$
U(z, 0)=\int_{0}^{\pi} V(1, \phi) Q^{*}(z, 0, \phi) \sin ^{2 \mu} \phi d \phi+U_{0},
$$

where

$$
\begin{gathered}
Q^{*}(z, 0, \phi)=\frac{2^{2 \mu} \Gamma^{2}(\mu+1)}{\pi \mu \Gamma(2 \mu)} \sum_{n=1}^{\infty} \frac{n+\mu}{n} \\
\cdot z^{n} C_{n-1}^{\mu+1}(\cos \phi) \sin \phi
\end{gathered}
$$

Using the generating relation (3.5) we have

$$
\frac{1}{\mu+1} \sum_{n=1}^{\infty} \frac{n+\mu}{n} z^{n} C_{n-1}^{\mu+1}(\cos \phi)=\int_{0}^{z} \frac{1-\zeta^{2}}{\left(1-2 \zeta \cos \phi+\zeta^{2}\right)^{\mu+2}} d \zeta .
$$

Further, $U(z, 0)=2^{1-2 \mu} \mu \Gamma(2 \mu) f(z)$, where $f$ is the $L_{\mu}^{*}$ associate of $V$. Thus we have the explicit integral representation for the inverse transform

$$
\begin{aligned}
f(z) & =\left(L^{*}\right)^{-1}(V) \\
& =a_{0}+\int_{0}^{\pi} V(1, \phi) H^{*}(z, \phi) \sin ^{2 \mu+1} \phi d \phi,
\end{aligned}
$$

where

$$
H^{*}(z, \phi)=\frac{2(\mu+1)}{\Gamma^{2}(\mu+1)} \int_{0}^{z} \frac{1-\zeta^{2}}{\left(1-2 \zeta \cos \phi+\zeta^{2}\right)^{\mu+2}} d \zeta
$$


4. Singularities. As is well known, if $u$ is a function which is harmonic in a simply connected region $\Omega$, then the conjugate $v$ of $u$ is also harmonic throughout $\Omega$, and thus $u=\operatorname{Re}(f)$ where $f(z)$ is analytic in $\Omega$. We next obtain the analogous result for generalized axisymmetric potentials, their conjugates, and $\mu$-pseudo analytic functions.

A function $U$ is said to be a regular generalized axisymmetric potential on a region $\Omega$ if it is a $C^{2}$ solution of equation (1.4) throughout $\Omega \backslash\{y=0\}$, and $\partial U(x, 0) \backslash \partial y=0$ for all $(x, 0) \in \Omega$. If $U$ is regular in a neighborhood of the origin, it has the expansion (2.2). The conjugate $V$ of $U$ is said to be regular in a region $\Omega$ if it is a $C^{2}$ solution of the system (1.5) throughout $\Omega \backslash\{y=0\}$ and $V(x, 0)=$ 0 for all $(x, 0) \in \Omega$. If $V$ is regular in a neighborhood of the origin, it has the expansion (2.5). We say that a region $\Omega$ is axiconvex if it is connected and $(x, y) \in \Omega$ implies $(x, \lambda y) \in \Omega$ for all $\lambda \in[-1,1]$.

The following result is owing to Gilbert [15]:

Let $U$ be a generalized axisymmetric potential and $f$ be its $L_{\mu}$ associate. Then $(x, \pm y)$ is a singularity of $U$ iff $x \pm i y$ is a singularity of $f$.

In particular, the axiconvex regions of regularity of $U$ and $f$ coincide (this result has also been obtained by Erdélyi [10]). Hence given $U$ regular on an axiconvex region $\Omega$, we have

$$
\begin{aligned}
V(r, \theta)=L_{k}^{*}(f)= & -\frac{i}{\mu \Gamma^{2}(\mu)} \int_{0}^{\pi} f(r \cos \theta+i r \sin \theta \cos t) \\
& \cdot \cos t \sin ^{2 \mu-1} t d t
\end{aligned}
$$

where $f$ is analytic on $\Omega$. Thus it is immediate that $V$ is of class $C^{2}$ throughout $\Omega$ and $V(x, 0)=0$. Further, using the identity

$$
\begin{aligned}
\int_{0}^{\pi} f^{\prime}(x+ & i y \cos t) \sin ^{2 \mu+1} t d t \\
& =-\frac{2 \mu i}{y} \int_{0}^{\pi} f(x+i y \cos t) \cos t \sin ^{2 \mu-1} t d t,
\end{aligned}
$$

it is easy to verify that $V$ is a solution of the system (1.5) throughout $\Omega \backslash\{y=0\}$. Thus $V$ is also regular on $\Omega$. We state this result as a theorem.

THEOREM 4.1. If $U$ is a generalized axisymmetric potential which is regular on an axiconvex region $\Omega$, then its conjugate $V$ is also regular on $\Omega$.

The following result is also immediate.

THEOREM 4.2. $F$ is $\mu$-pseudo analytic on an axiconvex region 
$\Omega$ if and only if its $\mathscr{L}_{\mu}$ associate $f$ is analytic throughout $\Omega$.

Proof. If $F$ is $\mu$-pseudo analytic on $\Omega$ then $U$ is regular on $\Omega$, and by Gilbert's result $f$ is analytic on $\Omega$. Conversely, if $f$ is analytic then $U=L_{t \prime}(f)$ is regular on $\Omega$, and by the last theorem its conjugate $V$ is regular on $\Omega$. Thus $F=U+i V=\mathscr{\rho}_{\mu}(f)$ is "pseudo analytic on $\Omega$.

5. Polynomial approximation. One of the most striking roles the transformation $u=\operatorname{Re}(f)$ plays in the study of harmonic functions lies in the theory of uniform polynomial approximation. While Fourier series techniques yield the uuiform harmonic polynomial $\sum_{k=0}^{n} \gamma^{k}\left(a_{n} \cos k \theta+b_{k} \sin k \theta\right)$ approximation to harmonic functions on a disk, they do not provide an adequate tool for dealing with such problems on more general regions. But in the latter case results obtain immediately using the operation Re to draw on the theory of uniform polynomial approximation for analytic functions, a rich and highly developed area.

Similarly, when dealing with more general elliptic equations, separation of variables and Fourier series expansion yields approximations on certain regions having a very simple geometry. Thus, for example, separation of variables for the equation (1.4) expressed in polar coordinates leads to the Gegenbauer polynomials, and the uniform approximation via partial sums of the series (2.2) to solutions which are regular on a disk. It is natural to expect similar approximations on regions having a more complicated geometry. Just as in the case of harmonic functions, using integral operators which generalize the operations $R e$ and Im, we shall obtain such results with ease.

Bers has developed a theory of "formal power" approximation for pseudo analytic functions which closely resembles the theory of polynomial approximation for analytic functions of a single complex variable. Recall that $u+i v$ is a $\mu$-pseudo analytic function of the second kind if and only if $u$ and $v$ satisfy the system (1.7). In this case, Bers' formal power $Z^{(n)}(\lambda, 0, z)$ of degree $n$, centered at 0 , with real coefficient $\lambda$ is given by

$$
\begin{aligned}
Z^{(n)}(\lambda, 0, z) & =\lambda Z^{(n)}(1,0, z) \\
& =\lambda \sum_{j=0}^{n}\left(\begin{array}{l}
n \\
j
\end{array}\right) x^{n-j} i^{j} Y^{(j)}(y),
\end{aligned}
$$

where

$$
Y^{(0)}(y)=1, Y^{(1)}(y)=\int_{0}^{y} \eta^{2 \mu} d \eta
$$




$$
Y^{(2)}(y)=2 \int_{0}^{y} \frac{Y^{(1)}(\eta)}{\eta^{2 \mu}} d \eta, \quad Y^{(3)}(y)=3 \int_{0}^{y} \eta^{2 \mu} Y^{(2)}(\eta) d \eta, \cdots
$$

(cf. [9, pp. 389-90]). The formal powers $Z^{(n)}(\lambda, 0, z)$ given above are $\mu$-pseudo analytic functions of the second kind. That is, writing

$$
Z^{(n)}(\lambda, 0, z)=u_{n}+i v_{n}, u_{n}, v_{n} \text { real, }
$$

then $u_{n}$ and $v_{n}$ satisfy the system (1.7). Since $\left[1, i y^{-2 \mu}\right]$ is a generating pair for $\mu$-pseudo analytic functions (see [9, p. 389]), the corresponding formal powers of the first kind are

$$
W^{(n)}(\lambda, 0, z)=u_{n}+i y^{-2 \mu} v_{n} .
$$

These powers are in fact homogeneous polynomials of degree $n$ in $x$ and $y$. For convenience let $W^{(k)}(z)=W^{(k)}(1,0, z)$. A $\mu$-pseudo analytic polynomial of degree $n$ is then a sum $\sum_{k=0}^{n} a_{k} W^{(k)}(z)$, where $a_{n} \neq 0$. The polynomials $W^{(n)}$ may also be computed as the $\mathscr{L}_{\mu}$ transforms of the powers $z^{n}$. That is,

$$
\begin{aligned}
& W^{(x)}\left(\frac{2^{2 \mu}}{\Gamma(2 \mu+1)}, 0, z\right)=\mathscr{L}_{\mu}\left(z^{n}\right) \\
& \quad=\frac{1}{\mu \Gamma^{2}(\mu)} \int_{0}^{\pi}(x+i y \cos t)^{n}(1+\cos t) \sin ^{2 \mu-1} t d t
\end{aligned}
$$

(cf. [8, pp. 175-177]). By either evaluating the integral (5.2) or proceeding inductively according to (5.1) the first few formal powers are easily computed as

$$
\begin{aligned}
W^{(0)}(z) & =1 \\
W^{(1)}(z) & =\frac{1}{2 \mu+1}[(2 \mu+1) x+i y] \\
W^{(2)}(z) & =\frac{1}{2 \mu+1}\left[(2 \mu+1) x^{2}-y^{2}+i 2 x y\right] \\
W^{(3)}(z) & =\frac{3}{(2 \mu+3)(2 \mu+1)} \\
\times & \left\{\frac{(2 \mu+3)(2 \mu+1)}{3} x^{3}-(2 \mu+3) x^{2} y^{2}+i\left[(2 \mu+3) x^{2} y-y^{3}\right]\right\} .
\end{aligned}
$$

Notice that $W^{(n)}(z) \rightarrow z^{n}$ as $\mu \rightarrow 0$.

Bers has obtained an analog of Runge's theorem regarding the uniform approximation of pseudo analytic functions by formal powers [4, p. 119]. We consider the rate of convergence of the approximating polynomials in the case of $\mu$-pseudo analytic functions.

Let $\mathrm{Cl}(\Omega)$ denote the closure of a region $\Omega$. We say $F$ is $\mu$ pseudo analytic on $\mathrm{Cl}(\Omega)$ if it is $\mu$-pseudo analytic throughout some 
larger region $\Omega^{\prime} \supset \Omega$.

THEOREM 5.1. Let $\Omega$ be a bounded axiconvex region. If $F$ is $\mu$-pseudo analytic on $\mathrm{Cl}(\Omega)$, then there exist $\mu$-pseudo analytic polynomials

$$
W_{n}(z)=\sum_{k=0}^{n} a_{k} W^{(k)}(z)
$$

such that

$$
\left\|F-W_{n}\right\| \leqq \frac{M}{R^{n}},
$$

where $\|\cdot\|$ denotes the sup norm over $\mathrm{Cl}(\Omega), M$ is a constant independent of $n$, and $R>1$.

Proof. By Theorem 4.2 the $\mathscr{L}_{\mu}$ associate $f$ of $F$ is analytic on $\mathrm{Cl}(\Omega)$. Thus there exist polynomials $P_{n}(z)=\sum_{k=0}^{n} b_{k} z^{k}$ such that

$$
\max _{z \in \mathrm{Cl}(\Omega)}\left|f(z)-p_{n}(z)\right|=\left\|f-p_{n}\right\| \leqq \frac{M}{R^{n}},
$$

where $R>1$ and $M$ is a constant independent of $n$ (see [25, pp. 75-76]). Now

$$
\begin{aligned}
\mathscr{L}_{\mu}\left[p_{n}(z)\right] & =\sum_{k=0}^{n} b_{k} \mathscr{L}_{\mu}\left(z^{k}\right) \\
& =\frac{2^{2 \mu}}{\Gamma(2 \mu+1)} \sum_{k=0}^{n} b_{k} W^{(k)}(z)
\end{aligned}
$$

are $\mu$-pseudo analytic polynomials of degree $n$-call them $W_{n}(z)$. Then

$$
\begin{aligned}
\left\|F-W_{n}\right\| & =\left\|\mathscr{L}_{\mu}\left(f-p_{n}\right)\right\| \\
& \leqq \frac{2 \sqrt{\pi}}{\Gamma(\mu+1) \Gamma(\mu+1 / 2)}\left\|f-p_{n}\right\| \\
& \leqq \frac{2 \sqrt{\pi}}{\Gamma(\mu+1) \Gamma(\mu+1 / 2)} \frac{M}{R^{n}} .
\end{aligned}
$$

While the general theory of pseudo analytic functions enjoys an analog of Runge's polynomial approximation theorem, with the exception of the preceding result, no information regarding the rate of convergence of the formal power approximants seems to have been obtained. When proceeding in analogy with classical function theory, severe difficulties are encountered owing to the fact that pseudo analytic functions do not form an algebra (i.e., products of 
pseudo analytic functions need not be pseudo analytic). Nevertheless, the preceeding theorem, as well as close analogies with function theory, suggests that formal power approximants to functions which are regular in the closure of a region may in general converge at a geometric rate.

We next obtain results which are more constructive in nature. It is possible to introduce analogs of the Faber polynomials. Recall that for a bounded simply connected $\Omega$, if $w=\phi(z)=z+a_{0}+a_{1} z^{-1}+\cdots$ maps the compliment of $\Omega$ onto the circular region $|w|>\rho$, then the Faber polynomials $f_{n}(z)$ of degree $n$ for $\Omega$ are defined by the generating relation

$$
\frac{t \psi^{\prime}(t)}{\psi(t)-z}=\sum_{n=0}^{\infty} f_{n}(z) t^{-n}
$$

where $\psi=\dot{\phi}^{-1}$ (see [22, p. 130]). We define

$$
F_{n}=\mathscr{L}_{\mu}\left(f_{n}\right)
$$

and call $F_{n}$ the $\mu$-pseudo analytic Faber polynomials for $\Omega$.

THEOREM 5.2. Let $\Omega$ be a bounded axiconvex region, $w=\phi(z)=$ $z+a_{0}+a_{1} z^{-1}+\cdots$ map the compliment of $\Omega$ onto the circular region $|w|>\rho$, and let $\psi=\phi^{-1}$. If $F(x, y)$ is $\mu$-pseudo analytic on the region $B_{R}$ bounded by $\Gamma_{R}=\{z:|\phi(z)|=R\}$, then $F$ can be expanded into a series of $\mu$-pseudo analytic Faber polynomials

$$
F(x, y)=\sum_{n=0}^{\infty} a_{n} F_{n}(x, y),
$$

where

$$
a_{n}=\frac{\mu \Gamma(2 \mu)}{2^{2 \mu} \pi i} \int_{|z|=r} F(\psi(z), 0) z^{-k-1} d z, \rho<r<R .
$$

Further

$$
\lim _{n \rightarrow \infty} \sup \left|a_{n}\right|^{1 / n}=1 / R,
$$

and the series (4.3) converges uniformly on $\mathrm{Cl}(\Omega)$ at a geometric rate.

Proof. Since $F$ is $\mu$-pseudo analytic on $B_{R}$, Theorem 3.2 insures its $\mathscr{L}_{\mu}$ associate $f$ is analytic on $B_{R}$. Thus

$$
f(\boldsymbol{z})=\sum_{n=0}^{\infty} a_{n} f_{n}(z),
$$

where $f_{n}$ are the Faber polynomials for $\Omega$, and 


$$
b_{\varkappa}=\frac{1}{2 \pi i} \int_{|z|=r} f(\psi(z)) z^{-k-1} d z, \rho<r<R
$$

(see [22, p. 138]). Also, $\left|b_{n}\right|^{1 / n} \rightarrow 1 / R$, and for $z \in \partial \Omega,\left|f_{n}(z)\right|^{1 / n} \rightarrow \rho$. Taking the $f /$ transform of (5.5) we have

$$
F(x, y)=\sum_{n=0}^{\infty} a_{n} F_{n}(x, y) .
$$

Let $\|\cdot\|$ denote the sup norm on $\mathrm{Cl}(\Omega)$. Since

$$
\left\|F_{n}\right\|=\left\|\mathscr{C}_{\mu}\left(f_{n}\right)\right\|_{1} \leqq c\left\|f_{n}\right\|,
$$

we have

$$
\lim _{n \rightarrow \infty}\left\|\boldsymbol{F}_{n}\right\|^{1 / n} \leqq \lim _{n \rightarrow \infty}\left\|f_{n}\right\|^{1 / n} \leqq \rho
$$

Since $\left|a_{n}\right|^{1 / n} \rightarrow R>\rho$, the series (5.6) converges uniformly on $\mathrm{Cl}(\Omega)$. Also restricting $F(x, y)=U(x, y)+i V(x, y)$ to the $x$-axis and analytically continuing to complex variables yields

$$
F(z, 0)=U(z, 0)=\frac{2^{2 \mu-1}}{\mu \Gamma(2 \mu)} f(z),
$$

where $f$ is the $\mathscr{L}_{\mu}$ associate of $F$. Thus the expression (5.4) obtains for the coefficients $a_{n}$.

It is also possible to obtain uniform polynomial approximation to $\mu$-pseudo analytic functions via polynomial interpolation. Let $\Omega$ be an axiconvex region and $C_{n}=\left\{z_{k}=x_{k}+i y_{k}\right\}_{k=0}^{n}$ be a set of $n+1$ points on $\partial \Omega$. Define

$$
V\left(C_{n}\right)=\operatorname{det}\left[W^{(k)}\left(z_{j}\right)\right]_{k, j=0}^{n},
$$

and

$$
V_{k}\left(z ; C_{n}\right)=\left.V\left(C_{n}\right)\right|_{z_{k}=z} \text {. }
$$

If $V\left(C_{n}\right) \neq 0$, and $F$ is any function defined on $\partial \Omega$, then

$$
L_{n}\left(z ; C_{n} ; F\right)=\sum_{k=0}^{n} F\left(z_{k}\right) \frac{V_{k}\left(z ; C_{n}\right)}{V\left(C_{n}\right)}
$$

is the unique $\mu$-pseudo analytic polynomial of degree $n$ which interpolates to $F$ on $C_{n}$. Further, it is easy to show that for $n=1,2,3 \cdots$ there exist $C_{n} \subset \partial \Omega$ such that $V\left(C_{n}\right) \neq 0$. The result is clearly true for $n=1$ i.e., if $C_{1}=\left\{z_{0}, z_{1}\right\}$ where $z_{0} \neq z_{1}$, then

$$
V\left(C_{1}\right)=\left|\begin{array}{ll}
1 & W^{(1)}\left(\boldsymbol{z}_{0}\right) \\
1 & W^{(2)}\left(z_{0}\right)
\end{array}\right|=x_{1}-x_{0}+\frac{i}{2 \mu+1}\left(y_{1}-y_{0}\right) \neq 0 .
$$


Suppose there exist $C_{n-1}=\left\{z_{0}, z_{1}, \cdots, z_{n-1}\right\} \subset \partial \Omega$ such that $V\left(C_{n-1}\right) \neq 0$. Then letting $C_{n}=\left\{z_{0}, \cdots, z_{n-1}, z_{n}\right\}$, we have on expanding $V\left(C_{n}\right)$ by the first row,

$$
V\left(C_{n}\right)=V\left(C_{n-1}\right) W^{(n)}\left(z_{n}\right)+b_{n-1} W^{(n-1)}\left(z_{n}\right)+\cdots+b_{0},
$$

where the coefficients $b_{k}$ are functions of $z_{0}, z_{1}, \cdots, z_{n-1}$. By hypothesis $V\left(C_{n-1}\right) \neq 0$, and thus $V\left(C_{n}\right)$ is a $\mu$-pseudo analytic polynomial of degree $n$ in $z_{n}$. Since the zeros of a nonconstant pseudo analytic function are isolated $\left[4\right.$, p. 18] we easily have a $z_{n} \in \partial \Omega$ such that $V\left(C_{n}\right) \neq 0$.

THEOREM 5.3. Let $\Omega$ be a bounded axiconvex region and $C_{n}^{*}$ maximize $\left|V\left(C_{n}\right)\right|$ over $\mathrm{Cl}(\Omega)$. Then

$$
\lim _{n \rightarrow \infty} L_{n}\left(z ; C_{n}^{*} ; F\right)=F(z)
$$

uniformly on $\mathrm{Cl}(\Omega)$ for every function $F$ which is $\mu$-pseudo analytic on $\mathrm{Cl}(\Omega)$. Further, the convergence is at a geometric rate, i.e.,

$$
\left\|F-L_{n}\right\| \leqq \frac{(n+2) M}{R^{n}}
$$

where $\|\cdot\|$ denotes the sup norm over $\Omega$ and the constants $M, R>1$ are as given in Theorem 5.1.

Proof. By Theorem 5.1 there exist $\mu$-pseudo analytic polynomials $p_{n}$ of degree $n$ such that

$$
\left\|F-p_{n}\right\| \leqq \frac{M}{R^{n}}
$$

Since $V\left(C_{n}^{*}\right) \neq 0$, the $\mu$-pseudo analytic polynomial of degree $n$ assuming $n+1$ given values at the points $z_{0}, z_{1}, \cdots, z_{n} \in C_{n}^{*}$ is unique. Thus

$$
L_{n}\left(z ; C_{n}^{*} ; p_{n}\right)=\sum_{k=0}^{n} p_{n}\left(z_{k}\right) \frac{V_{k}\left(z ; C_{n}^{*}\right)}{V\left(C_{n}^{*}\right)} \equiv p_{n}(z),
$$

and

$$
\begin{aligned}
\mid F(z)- & L_{n}\left(z ; C_{n}^{*} ; F\right)|\leqq| F(z)-p_{n}(z)|+| p_{n}(z)-L_{n}\left(z ; C_{n}^{*} ; F\right) \mid \\
& =\left|F(z)-p_{n}(z)\right|+\left|\sum_{k=0}^{n}\left[p_{n}\left(z_{k}\right)-F\left(z_{k}\right)\right] \frac{V_{k}\left(z ; C_{n}^{*}\right)}{V\left(C_{n}^{*}\right)}\right| \\
& \leqq \frac{M}{R^{n}}\left(1+\sum_{k=0}^{n} \frac{V_{k}\left(z ; C_{n}^{*}\right)}{V\left(C_{n}^{*}\right)}\right) \\
& \leqq \frac{(n+2) M}{R^{n}}
\end{aligned}
$$


By taking real and imaginary parts of the $\mu$-pseudo analytic functions involved, the previous theorems yield results regarding the uniform approximation to solutions of the elliptic equations (1.4) and (1.6) by polynomial solutions of the equations. For further results regarding the approximation of solutions of equation (1.4) see [11] and [13].

\section{REFERENCES}

1. S. Agmon and L. Bers, The expansion theorem for pseudo-analytic functions, Proc. Amer. Math. Soc., 3 (1952), 757-764.

2. R. Askey, Norm inequalities for some orthogonal series, Bull. Amer. Math. Soc., 72 (1966), 808-823.

3. S. Bergman, Integral Operators in The Theory of Linear Partial Differential Equations, Springer-Verlag, 1969.

4. L. Bers, Theory of Pseudo-Analytic Functions, New York University (Lecture Notes), 1953.

5 . An outline of the theory of pseudoanalytic functions, Bull. Amer. Math. Soc., 62 (1956), 291-331.

6. — Formal powers and power series, Comm. Pure Appl. Math., 9 (1956), 693-711.

7. - The expansion theorem for sigma-monogenic functions, Amer. J. Math., 72 (1950), 705-712.

8. L. Bers and A. Gelbart, On a class of differential equations in mechanics of continua, Quaterly of Applied Mathematics, 1 (1943), 168-188.

9. R. Courant and D. Hilbert, Methods of Mathematical Physics, Vol. 2, Intersciences, 1962.

10. A. Erdélyi, Singularities of generalized axially symmetric potentials, Comm. Pure Appl. Math., 9 (1956), 403-414.

11. A. Fryant, Interpolation and approximation of generalized axisymmetric potentials, SIAM Journal of Math. Anal., 9 (1978), 906-914.

12. - Extension of Privaloff's theorem to ultraspherical expansions, Proc. Amer. Math. Soc., 71 (1978), 49-53.

13. - Growth and complete sequences of generalized axisymmetric potentials, J. Approximation Theory, 19 (1977), 361-370.

14. R. Gilbert, Function Theoretic Methods in Partial Differential Equations, Academic Press, 1969.

15. - On the singularities of generalized axially symmetric potentials, Arch. Rational Mech. Anal., 6 (1960), 171-176.

16. B. Golusin, Geometric Theory of Functions of a Complex Variable, Translations of Mathematical Monographs Vol. 26, Amer. Math. Soc., 1969.

17. G. Hardy and J. Littlewood, Some properties of fractional integrals II, Math. Z., 34 (1931), 404-439.

18. A. Heins, Axially symmetric boundary value problems, Bull. Amer. Math. Soc., 71 (1965), 787-808.

19. B. Muckenhaupt and E. Stein, Classical expansions and their relation to conyugate harmonic functions, Trans. Amer. Math. Soc., 118 (1965), 17-92.

20. I. Pravaloff, Sur les fonctiones conjugees, Bull. Soc. Math. France, 44 (1916), 100-103.

21. S. Poisson, Sur l'integration des equations lineaires aux differences partielles, J. Ecole Roy. Polytechnique, 12 (1823), 215-248.

22. V. Smirnov and N. Lebedev, Functions of a Complex Variable, MIT Press, 1968.

23. F. Tricomi, Vorlesungen Uber Orthogonalreihen, Springer-Verlag, 1955.

24. I. Vekua, Generalized Analytic Functions, Addison-Wesley, 1962.

25. J. Walsh, Interpolation and approximation by rational functions in the complex 
domain, Amer. Math. Soc., 1969.

26. A. Weinstein, Generalized axially symmetric potential theory, Bull. Amer. Math. Soc., 59 (1953), 20-38.

Received October 9, 1978. Supported by a grant from the U.S. Naval Academy Research Council.

Department of the Navy

United States Naval Academy

Annapolis, MD 21402 



\section{PACIFIC JOURNAL OF MATHEMATICS}

\section{EDITORS}

DONALD BABBITT (Managing Editor)

University of Galifornia

Los Angeles, California 90024

Hugo RossI

University of Utah

Salt Lake City, UT 84112

C. C. MOORE AND ANDREW OGG

University of California

Berkeley, CA 94720

\section{J. DugundjI}

Department of Mathematics University of Southern California

Los Angeles, California 90007

R. Finn AND J. Milgram

Stanford University

Stanford, California 94305

\section{ASSOCIATE EDITORS}

R. ARENS

E. F. BECKENBACH

B. H. NeUmanN

F. WOLF

K. YosHIDA

\section{SUPPORTING INSTITUTIONS}

UNIVERSITY OF ARIZONA

UNIVERSITY OF BRITISH COLUMBIA

CALIFORNIA INSTITUTE OF TECHNOLOGY

UNIVERSITY OF CALIFORNIA

MONTANA STATE UNIVERSITY

UNIVERSITY OF NEVADA, RENO

NEW MEXICO STATE UNIVERSITY

OREGON STATE UNIVERSITY
UNIVERSITY OF OREGON

UNIVERSITY OF SOUTHERN CALIFONIA

STANFORD UNIVERSITY

UNIVERSITY OF HAWAII

UNIVERSITY OF TOKYO

UNIVERSITY OF UTAH

WASHINGTON STATE UNIVERSITY

UNIVERSITY OF WASHINGTON 


\section{Pacific Journal of Mathematics}

\section{Vol. 94, No. 1 \\ May, 1981}

Willy Brandal, Conditions for being an FGC domain $\ldots \ldots \ldots \ldots \ldots \ldots \ldots$

Allan Calder and Frank Williams, Incompressibility of maps and the

homotopy invariance of Čech cohomology $\ldots \ldots \ldots \ldots \ldots \ldots \ldots \ldots \ldots$

Jacques Chaumat, Quelques propriétés du couple d'espaces vectoriels

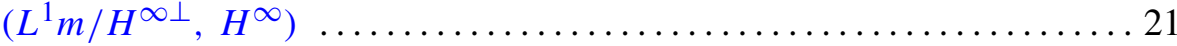

Manfred Droste and Rüdiger Göbel, Products of conjugate permutations . . 47

Jean Esterle, Rates of decrease of sequences of powers in commutative

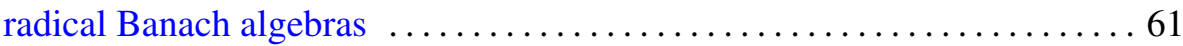

Allan Fryant, Ultraspherical expansions and pseudo analytic functions . . . 83

John Hannah, Homogenization of regular rings of bounded index. II . . . . . 107

Shigeru Haruki, On the theorem of S. Kakutani-M. Nagumo and J. L. Walsh

for the mean value property of harmonic and complex polynomials $\ldots .113$

Hugh M. Hilden, Representations of homology 3-spheres $\ldots \ldots \ldots \ldots \ldots 125$

Craig Huneke, A characterization of locally Macaulay completions . . . . . 131

Takesi Isiwata, Closed ultrafilters and realcompactness ................. 139

Joseph Weston Kitchen, Jr. and David A. Robbins, Tensor products of

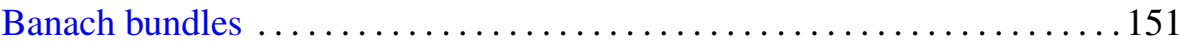

Allan J. Kroopnick, Note on bounded $L^{p}$-solutions of a generalized Liénard

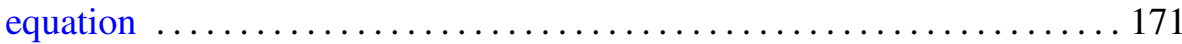

Ajay Kumar and Ajit Kaur Chilana, Spectral synthesis in products and quotients of hypergroups

Charles Livingston, Homology cobordisms of 3-manifolds, knot

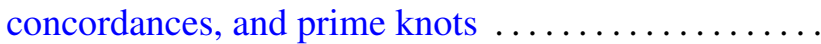

Hans Opolka, Projective representations of finite groups in cyclotomic fields

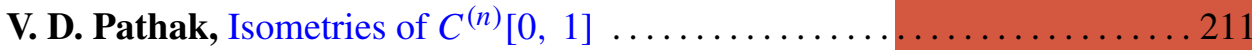

Mark Allan Pinsky, On the spectrum of Cartan-Hadamard manifolds . . . . . 2223

Judith Roitman, The number of automorphisms of an atomic Boolean

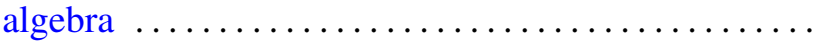

Kai Wang, Locally smooth torus group actions on integral cohomology complex projective spaces 